\title{
Food cravings and the effects of left prefrontal repetitive transcranial magnetic stimulation using an improved sham condition
}

\section{Kelly S. Barth ${ }^{1 *}$, Sofia Rydin-Gray ${ }^{2}$, Samet Kose ${ }^{3}$, Jeffrey J. Borckardt' ${ }^{1}$ Patrick M. O'Neil' , Darlene Shaw', Alok Madan', Amanda Budak ${ }^{4}$ and Mark S. George ${ }^{1}$}

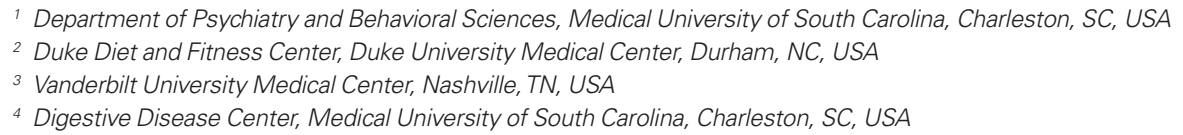

\section{Edited by:}

Darin Dougherty, Massachusetts

General Hospital, USA

\section{Reviewed by:}

Tal Herbsman, University of Wisconsin, USA

Tarek Rajii, Centre for Addiction and Mental Health, Canada

Paul Holtzheimer, Emory University

School of Medicine, USA

Patrick John Marsh, University of

South Florida, USA

*Correspondence:

Kelly S. Barth, Department of Psychiatry and Behavioral Sciences,

Medical University of South Carolina,

1-South, 67 President Street,

Charleston, SC 29425, USA.

e-mail: stephen@musc.edu
This study examined whether a single session of repetitive transcranial magnetic stimulation (rTMS) of the left prefrontal cortex (PFC) would inhibit food cravings in healthy women who endorsed frequent food cravings. Ten participants viewed images of food and completed ratings for food cravings before and after receiving either real or sham rTMS over the left PFC $(10 \mathrm{~Hz}$, $100 \%$ resting motor threshold, 10 s-on, 20 s-off for 15 min; 3000 pulses). Sham-TMS was matched with realTMS with respect to perceived painfulness of the stimulation. Each participant received both real and sham rTMS in random order and were blind to the condition in a within-subject cross-over design. With an improved sham control condition, prefrontal rTMS inhibited food cravings no better than sham rTMS. The mild pain from the real and sham rTMS may distract or inhibit food craving, and the decreased craving may not be caused by the effect of rTMS itself. Further studies are needed to elucidate whether rTMS has any true effects on food craving and whether painful stimuli inhibit food or other cravings. A sham condition which matches the painfulness is important to understand the true effects of TMS on behaviors and diseases.

Keywords: transcranial magnetic stimulation, TMS, food cravings, inhibition

\section{INTRODUCTION}

While the role of the prefrontal cortex $(\mathrm{PFC})$ in regulating cravings remains somewhat unclear, frontal cortical areas appear to be involved in integrating incoming sensory information (such as sights, smells, and sounds) with affective/emotional information in the brain, and may be involved in regulating emotional reactions to various stimuli (Alexander et al., 1986; Lorenz et al., 2003). The dorsolateral PFC may become activated when an individual is presented with cues that trigger reward memories associated with certain consumptive behaviors (Anton, 1999). For example, one fMRI study found that when alcoholic subjects were presented with alcohol-related cues, there was greater activation in the left PFC and anterior thalamus compared to when they viewed non-alcoholic cues (George et al., 2001). Other studies on bulimia and drug cravings have identified hyperactivity in the orbitofrontal cortex and anterior cingulate cortex associated with increased cravings ratings (Goldstein and Volkow, 2002; Uher et al., 2004).

Although very few studies have attempted to directly manipulate activation of brain structures that might be involved in cravings, TMS allows researchers the opportunity to selectively activate or inhibit different brain structures that might play a role in craving behaviors. One such study found that fast repetitive transcranial magnetic stimulation (rTMS) of the left dorsolateral PFC decreased cravings to smoke tobacco in smokers (Johann et al., 2003).
This finding, combined with prior neuroanatomical work, suggest that the PFC may be a reasonable preliminary TMS cortical target for potentially inhibiting food cravings.

There is little research on the effects of TMS on food cravings in humans. An initial small study by Uher et al. (2005) found that rTMS to the left PFC may inhibit increases in food cravings when compared with sham rTMS. There were several limitations to this study. Emerging evidence suggests some individuals experience rTMS to be mildly to moderately painful (Borckardt et al., 2006a). First of all, the sham system used in that study did not produce any sensations, so the painfulness of the active rTMS may have altered cravings and the observed effects may be unrelated to left prefrontal activation. Secondly, the number of pulses delivered (1000) may have been insufficient to maximize the treatment effects; a higher dose of rTMS may lead to less ambiguous effects (Borckardt et al., 2006a, 2008a). Finally, because that study used a between-subject design with a small sample $(N=28)$, the inter-subject variability may have limited the magnitude of the observed effect. In contrast to a small randomized controlled trial which found no benefit of rTMS in bingeing or purging behaviors in bulimic subjects (Walpoth et al., 2008), a subsequent study by Van den Eynde et al. (2010) using active rTMS vs. sham in bulimic subjects found significant results favoring rTMS in food craving. In this study, however, the groups were not successfully blinded, with significantly more of the active rTMS subjects guessing their assignment correctly 
compared with sham rTMS. This further highlights the importance of developing a sham rTMS condition that replicates the same level of pain as the active rTMS condition.

In this study, we matched discomfort to the scalp and face associated with active rTMS using an improved sham rTMS system (Borckardt et al., 2008b), and we investigated the effects of a higher dose of rTMS on food cravings, given the positive analgesic effects found in a previous postoperative trial using a higher dose of TMS (Borckardt et al., 2008a). We also employed a within-subjects, cross-over design to allow each participant to receive both real and sham rTMS, minimizing error variability between conditions. It was hypothesized that active rTMS would result in significantly less reported food cravings than would sham rTMS.

\section{MATERIALS AND METHODS PARTICIPANTS}

Twenty-nine individuals were screened for inclusion/exclusion criteria and food craving using the Food Craving Inventory (White et al., 2002). Participants had to endorse cravings at least three times per week during the past month for at least one of the following food groups: sweets, fast food fats, high fats, and/or carbohydrates. Inclusion criteria were: women age 18-40; BMI 20-40; and capable of giving consent. Exclusion criteria were: personal or family history of seizures, taking medication that lowers seizure threshold, suicidality, implanted metal devices, pregnancy, history of brain surgery or loss of consciousness, type I Diabetes, or currently in a weight loss program.

A total of 29 individuals were screened for the study. Eleven individuals were excluded based on the food craving questions, pregnancy status, or biological family members with epilepsy or seizure disorder. Seven individuals qualified but decided to not participate because of fears related to the TMS procedures. One individual discontinued her participation after receiving real TMS for $3 \mathrm{~min}$ due to physical discomfort. Ten healthy adult women completed the study and provided informed consent. They were asked not to eat $3 \mathrm{~h}$ prior to the appointments (allowed normal amounts of liquid) and were reimbursed $\$ 25.00$ for participation. The Institutional Review Board of the Medical University of South Carolina approved this study. Table 1 describes participant characteristics.

\section{Determining motor threshold and locating cortical targets}

Resting motor threshold (rMT) was determined using a Neuronetics ${ }^{\circledast}$ Model 3600 with a solid focal coil TMS machine (Neuronetics, Malvern, PA, USA) starting with $40 \%$ of the machine output and $0.5 \mathrm{~Hz}$ stimu-

Table 1 | Means and standard deviations for participant characteristics and scores on the BDI.

\begin{tabular}{lrr}
\hline $\mathbf{N = 1 0}$ & Mean & SD \\
\hline Age & 28.3 & 6.5 \\
Body mass index & 27.8 & 8.0 \\
Weight & 165.8 & 50.3 \\
Weeks since last menstrual cycle & 2.2 & 0.59 \\
BDI & 8.1 & 5.7 \\
Hours since last meal real TMS & 6.7 & 3.8 \\
Hours since last meal sham-TMS & 5.9 & 3.7
\end{tabular}

lus frequency. The coil was positioned over the area of the skull corresponding to the motor cortex, adjusted until each pulse resulted in isolated movement of the right thumb (abductor pollicis brevis - APB), and then adjusted for the lowest intensity that reliably produced thumb movement. Parameter Estimation by Sequential Testing (Borckardt et al., 2006b) was used to estimate the output necessary to produce thumb movement $50 \%$ of the time. The position of the coil used for rMT assessment was identified as the motor cortex target (M1). Localization of left PFC was defined as $5 \mathrm{~cm}$ anterior to M1 along a parasagittal line (George et al., 1995, 1996, 1997; Uher et al., 2005).

\section{Real rTMS condition}

Participants received $10 \mathrm{~Hz}$, at 100\% rMT, 10 s-on, and 20 s-off for 15 min, producing 3000 pulses in a single 15 -min session. These settings were associated with no unanticipated complications in a recent TMS trial (Borckardt et al., 2006a, 2008a).

\section{Sham-TMS procedures}

After rMT determination and PFC cortex localization, participants were fitted with two electrodes on the scalp just below the hairline. Electrodes were connected to an Epix VT ${ }^{\oplus}$ Transcutaneous Electrical Nerve Stimulation Device (Empi; St. Paul, MN, USA) with adjustable output amplitude and powered by a 9-V battery. For each participant, the electrical sham system was turned off, and a series of 1-s trains of real $10 \mathrm{~Hz}$ prefrontal rTMS at $100 \%$ of rMT was delivered. Participants rated the painfulness of each train using an 11-point rating scale ( $0=$ no pain, $10=$ worst pain). After obtaining a reliable estimate of each participant's pain-rating associated real rTMS (100\% rMT, $10 \mathrm{~Hz})$, the electrical current of the sham system using a sham rTMS coil was titrated to a level matching participants' ratings of real rTMS. All participants underwent sham titration procedures before each rTMS session and wore the scalp electrodes in both conditions. We used $10 \mathrm{~Hz}$ TMS, $10 \mathrm{~s}$-on, $20 \mathrm{~s}$-off for $20 \mathrm{~min}$ in both real and sham conditions. Sham-TMS scalp discomfort was matched to that of real TMS at $100 \%$. See Borckardt et al. (2008b) for more details.

\section{PROCEDURES}

Participation entailed two 60-min rTMS visits on two consecutive Fridays during the luteal phase of the participants' menstrual cycle (a time of increased food cravings; Davidsen et al., 2007). Written informed consent, time of last meal, BMI, and Beck Depression Inventory (Beck et al., 1996) were obtained. A series of standardized color food images from the International Affective Picture System (IAPS) were presented on a computer screen. Two-dimensional food pictures are widely used in food craving studies and are associated with unique cortical activation relative to non-food item pictures (Killgore et al., 2003). Twenty-three images of highly palatable foods were presented in random order. While viewing the food images, participants used a computerized visual analog scale (CVAS) to rate how much they "would like to eat each food right now" if available to them. After viewing the images, participants received 15 min of real or sham rTMS, using the parameters described above. After 15 min of real or sham rTMS, participants viewed the images again and rated their cravings. At each visit, participants were blind to the rTMS condition (real or sham) and the order was randomized. 


\section{DATA ANALYSES}

The $2 \times 2$ repeated-measure analysis of variance (ANOVA) and analysis of covariance (ANCOVA) models were used to assess the effects of time (pre- and post-rTMS) and condition (real vs. sham rTMS) on food craving ratings. The ANCOVA was run to examine the effects of time and condition on cravings after controlling for time since participants last ate.

\section{RESULTS}

\section{VALIDITY OF THE SHAM-TMS SYSTEM}

At the end of the study, participants were asked to guess which rTMS session was real and which was sham. Only $40 \%$ of participants guessed correctly $(p=0.27)$, suggesting that the sham condition was reasonably matched to real TMS with respect to face and scalp sensations.

\section{THE RELATIONSHIP AMONG CRAVINGS AND TMS CONDITION}

Analysis of variance results revealed that cravings were significantly lower following rTMS regardless of whether it was real or sham $[F(1,9)=15.91, p=0.003]$. The condition main effect $[F(1,9)=1.13$, ns $]$ and the time by condition interaction were not significant $[F(1,9)=0.13$, ns $]$. There was no significant difference in hours since last ate between sham and real TMS conditions $[t(9)=0.52, \mathrm{~ns}]$, but when we controlled for hours since participants last ate, no effects were observed for time $[F(1,8)=2.97$, ns], condition $[F(1,8)=1.94$, ns $]$, or the time by condition interaction $[F(1,8)=2.07, n s]$. Figure 1 displays the changes in cravings (estimated marginal means) for real and sham rTMS after controlling for hours since last meal. Change in cravings was slightly larger in the sham-TMS group, but the effect size (Cohen's $d$ ) was very small $(d=0.09)$ in favor of sham-TMS.

\section{DISCUSSION}

Repetitive transcranial magnetic stimulation was associated with a significant decrease in food cravings regardless of whether participants received real or sham rTMS. When controlling for hours since participants last ate, no effects were observed for rTMS on food cravings. Further, the mean food craving ratings do not suggest any trend toward a potential rTMS effect.

Partially consistent with the Uher et al. (2005) findings, fast left prefrontal rTMS was associated with significant change in food craving ratings; however, unlike the Uher et al. (2005) study, shamTMS was also associated with significant change in food craving ratings. One possible explanation is that the improved sham-TMS condition produced a comparable amount of pain as the real rTMS condition, and pain, rather than prefrontal activation, may have inhibited food cravings. Comparable pain may have also increased the likelihood that participants were truly blind to the condition. With each participant acting as his/her own control and randomized to real and sham rTMS sessions, potential carry-over effects were minimized.

There are several limitations to this study. The small sample size raises threats to external validity. However, the participants served as their own control for subjective food cravings when these cravings were examined on separate visits. The study may have been underpowered. However, the power was similar to that of the previous study, where a significant TMS effect was found, and the small effect size in this study would not suggest that a larger sample would detect significant differences. Finally, the rTMS operator knew the status of the participant, and subtle behavioral cues may have influenced outcomes in this single-blind paradigm. Future studies should consider a truly double-blind paradigm as has been developed for TMS depression trials. This

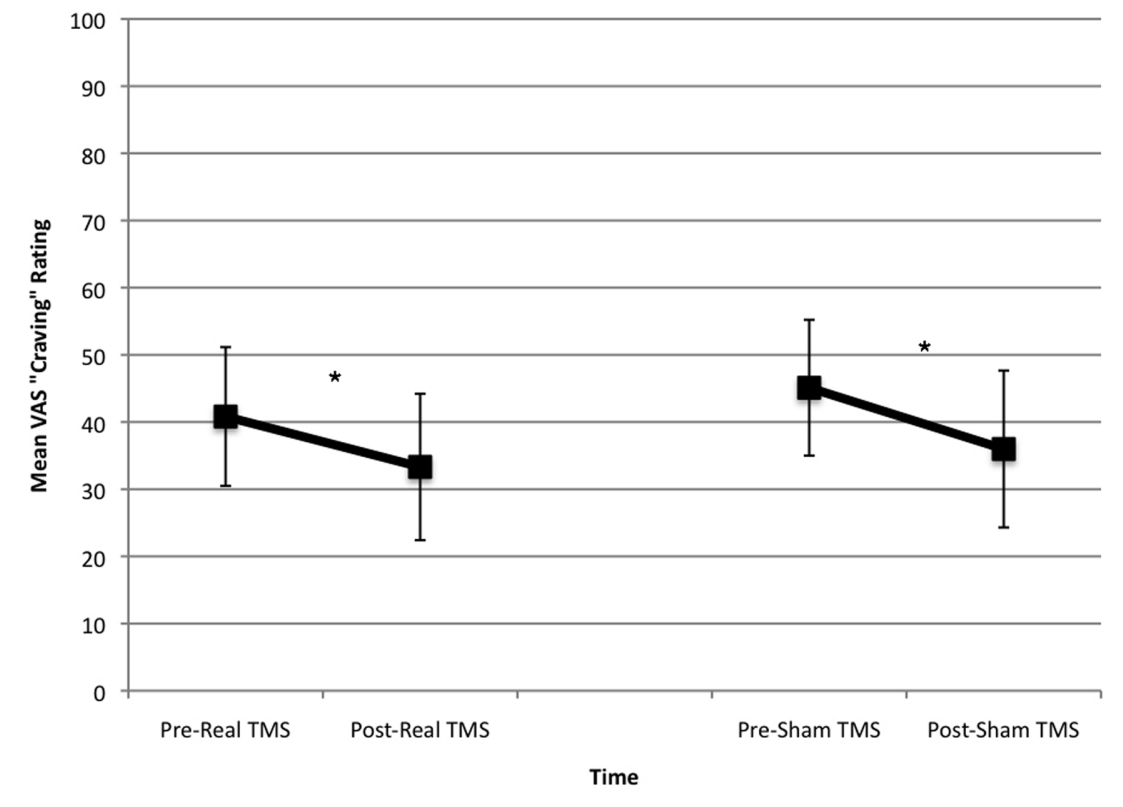

FIGURE 1 | Changes in cravings ratings in real and sham-TMS conditions. There was a significant drop in cravings in both the real-and sham-TMS conditions, with no difference between conditions before or after controlling for hours since last meal. 
study delivered rTMS at 100\% MT whereas the Uher study used rTMS at $110 \%$ MT. Because the "dose" of the TMS was higher in this study (3000 vs. 1500 pulses), we used the lower MT\% to ensure that we were using settings that had been previously been shown to be safe and effective. It is possible that this led to the decreased effectiveness in this study. One major limitation is that localization of the DLPFC may not have been accurate and could have contributed to the negative findings, and an MRI-guided method to localize the DLPFC would have been more accurate. However, the method described in this paper to localize the DLPFC has been used with good results in numerous previous trials and was more cost-effective for this pilot study.

\section{REFERENCES}

Alexander, G. E., DeLong, M. R., and Strick, P. L. (1986). Parallel organization of functionally segregated circuits linking basal ganglia and cortex. Annu. Rev. Neurosci. 9, 357-381.

Anton, R. F. (1999). What is craving? Models and implications for treatment. Alcohol Res. Health 23, 165-173.

Beck, A. T., Steer, R. A., and Brown, G. K. (1996). BDI-II, Beck Depression Inventory: Manual, 2nd Edn. San Antonio, TX: Psychological Corporation.

Borckardt, J. J., Reeves, S. T., Weinstein, M., Smith, A. R., Shelley, N., Kozel, F. A., Byrne, K. T., Morgan, K., and George, M. S. (2008a). Significant analgesic effects of one session of postoperative left prefrontal cortex repetitive transcranial magnetic stimulation: a replication study. Brain Stimul. 1, 122-127.

Borckardt, J. J., Walker, J., Branham, R. K., Rydin-Gray, S., Hunter, C., Beeson, H., Reeves, S. T., Madan, A., Sackeim, H., and George, M. S. (2008b). Development and evaluation of a portable sham TMS system. Brain Stimul. 1, 52-59.

Borckardt, J. J., Weinstein, M., Reeves, S. T., Kozel, F. A., Nahas, Z., Smith, A. R., Byrne, K. T., Morgan, K., and George, M. S (2006a). Postoperative left prefrontal repetitive transcranial magnetic stimulation reduces patient controlled analgesia use. Anesthesiology 105, 557-562.

Borckardt, J. J., Nahas, Z., Koola, J., and George, M.S. (2006b). Estimating resting motor thresholds in TMS research and practice: a computer simulation evaluation of best methods. J. ECT 22, 169-175.

Davidsen, L., Vistisen, B., and Astrup, A. (2007). Impact of menstrual cycle on determinants of energy balance: a putative role in weight loss attempts. Int. J. Obes. (Lond.) 31, 1777-1785.

George, M. S., Anton, R. F., Bloomer, C., Teneback, C., Drobes, D. J., Lorberbaum, J. P., Nahas, Z., and Vincent, D. J. (2001). Activation of prefrontal cortex and anterior thalamus in alcoholic subjects on exposure to alcohol-specific cues. Arch. Gen. Psychiatry 58, 345-352.

George, M. S., Wassermann, E. M., Kimbrell, T. A., Little, J. T., Williams, W. E., Danielson, A. L., Greenberg, B. D., Hallett, M., and Post, R. M. (1997). Mood improvement following daily left prefrontal repetitive transcranial magnetic stimulation in patients with depression: a placebo-controlled crossover trial. Am. J. Psychiatry 154, 1752-1756.

George, M. S., Wassermann, E. M., Williams, W. A., Callahan, A., Ketter, T., Basser, P., Hallett, M., and Post, R. M. (1995). Daily repetitive transcranial magnetic stimulation (rTMS) improves mood in depression. Neuroreport 6, 1853-1856.

George,M.S., Wassermann,E.M., Williams, W. A., Steppel, J., Pascual-Leone, A., Basser, P., Hallett, M., and Post, R. M. (1996). Changes in mood and hormone levels after rapid-rate transcranial magnetic stimulation (rTMS) of the prefrontal cortex. J. Neuropsychiatry Clin. Neurosci. 8, 172-180.

Goldstein, R.Z., and Volkow, N. D. (2002). Drug addiction and its underlying neurobiological basis: neuroimaging evidence for the involvement of the frontal cortex. Am. J. Psychiatry 159, 1642-1652.

Johann, M., Wiegand, R., Kharraz, A., Bobbe, G., Sommer, G., Hajak, G., Wodarz, N., and Eichhammer, P. (2003). Repetitive transcranial magnetic stimulation in nicotine dependence. Psychiatr. Prax. 30, 129-131.

Killgore, W. D. S., Young, A. D., Femia, L. A., Bogorodzki, P., Rogwska, J., and

More studies are needed to examine the true effects of rTMS on food craving. As the PFC may down-regulate emotional and motivational reactions to stimuli (Goldstein and Volkow, 2002; Lorenz et al., 2003; Uher et al., 2004), in this instance, the emotional reaction to painful stimuli may have inhibited craving and not the effects of rTMS. A follow-up study looking at the VAS scalp painratings before and after the TMS intervention would also answer the question raised in this paper about whether pain intensity is correlated with decreased craving.

\section{ACKNOWLEDGMENTS}

Berry Anderson, Minnie Dobbins, Teresa Garner.

Yurgelun-Todd, D.A. (2003). Cortical and limbic activation during viewing of high versus low-calorie foods. Neuroimage 1, 1381-1394.

Lorenz, J., Minoshima, S., and Casey, K. L. (2003). Keeping pain out of mind: the role of the dorsolateral prefrontal cortex in pain modulation. Brain 126 1079-1091.

Uher, R., Murphy, T., Brammer, M. J., Dalgleish, T., Phillips, M. L., and Ng, V. W. (2004). Medial prefrontal cortex activity associated with symptom provocation in eating disorders. Am. J. Psychiatry 161, 1238-1246.

Uher, R., Yoganathan, D., Mogg, A., Eranti, S. V., Treasure, J., Campbell, I. A., McLoughlin, D. M., and Schmidt, U. (2005). Effect of left prefrontal repetitive transcranial magnetic stimulation on food craving. Biol. Psychiatry 58, 840-842.

Van den Eynde, F., Claudino, A. M., Mogg, A., Horrell, L., Stahl, D., Ribeiro, W., Uher, R., Campbell, I., and Schmidt, U. (2010). Repetitive transcranial magnetic stimulation reduces cue-induced food craving in bulimic disorders. Biol. Psychiatry 67, 793-795.

Walpoth, M., Hoertnagl, C., MangwethMatzek, B., Kemmler, G., Hinterhölzl, J., Conca, A., and Hausmann, A. (2008). Repetitive transcranial magnetic stimulation in bulimia nervosa: preliminary results of a single-centre, randomised, double-blind, shamcontrolled trial in female outpatients. Psychother. Psychosom. 77, 57-60.

White, M. A., Whisenhunt, B. L. Williamson, D.A., Greenway, F. L., and Netemeyer, R. G. (2002). Development and validation of the food craving inventory. Obes. Res. 10, 107-114.

Conflict of Interest Statement: Dr. Borckardt receives research funding from the National Institute for Neurological Disorders and Stroke at NIH, Cyberonics Inc., and the Neurosciences Institute at
MUSC. Dr. George has received research funding from the National Institute for Mental Health, NIDA, and NIAAA at NIH, Force Protection, Jazz Pharmaceuticals, GlaxoSmithKline, and Brainsway. $\mathrm{He}$ is a consultant for Brainsonix, Brainsway, Cephos, Cyberonics, Neuronetics, Neuropace, Neostim, and PureTech Ventures. He has no equity ownership in any device or pharmaceutical company. Dr. Nahas reports having received consulting fees from Neuronetics Inc. and Cyberonics Inc; research funding from National Institute on Mental Health (NIMH), National Alliance of Research for Schizophrenia and Depression, Hope for Depression Research, Neuronetics Inc., Cyberonics Inc., Medtronic Inc., Brainsway and Integra. Dr. O’Neill receives research funding from Weight Watchers International, Orexigen Therapeutics, Merck Inc., BMS, and Arena Pharmaceuticals. He is on an advisory board for Orexigen Therapeutics and has consulted for Merck.

Received: 17 November 2010; paper pending published: 12 January 2011; accepted: 22 February 2011; published online: 14 March 2011.

Citation: Barth KS, Rydin-Gray S, Kose S, Borckardt JJ, O'Neil PM, Shaw D, Madan A, Budak A and George MS (2011) Food cravings and the effects of left prefrontal repetitive transcranial magnetic stimulation using an improved sham condition. Front. Psychiatry 2:9. doi: 10.3389/ fpsyt.2011.00009

This article was submitted to Frontiers in Neuropsychiatric Imaging and Stimulation, a specialty of Frontiers in Psychiatry. Copyright (c) 2011 Barth, Rydin-Gray, Kose, Borckardt, O'Neil, Shaw, Madan, Budak and George. This is an open-access article subject to an exclusive license agreement between the authors and Frontiers Media SA, which permits unrestricted use, distribution, and reproduction in any medium, provided the original authors and source are credited. 